Article

\title{
Computational Evaluation for Age-Dependent Material Nonlinear Behavior of Aortic Wall Tissue on Abdominal Aortic Aneurysms
}

\author{
Chung-Won Lee ${ }^{1,2}$, Up Huh ${ }^{1,2}$, Ji-Hun You ${ }^{3}$, Chi-Seung Lee ${ }^{2,4,5}\left(\right.$, Ki-Hoon Kim ${ }^{6}$, \\ Chan-Hee Song ${ }^{4}$, Jei-Pil Wang ${ }^{7}$ and Dong-Man Ryu ${ }^{2,4, *}$ \\ 1 Department of Thoracic and Cardiovascular Surgery, School of Medicine, Pusan National University, \\ Busan 49241, Korea; vasculardoctorlee@gmail.com (C.-W.L.); tymfoo82@gmail.com (U.H.) \\ 2 Biomedical Research Institute, Pusan National University Hospital, Busan 49241, Korea; \\ victorich@pusan.ac.kr \\ 3 Department of Mechanical Engineering, Pukyong National University, Busan 48513, Korea; \\ 940807n@naver.com \\ 4 Department of Biomedical Engineering, School of Medicine, Pusan National University, Busan 49241, Korea; \\ chsong0125@pusan.ac.kr \\ 5 School of Medicine, Pusan National University, Busan 49241, Korea \\ 6 Department of Mechanical Automotive Engineering, Pukyong National University, Busan 48513, Korea; \\ gasma21@naver.com \\ 7 Department of Metallurgical Engineering, Pukyong National University, Busan 48513, Korea; \\ jpwang@pknu.ac.kr \\ * Correspondence: babopanda@pusan.ac.kr; Tel.: +82-51-240-6867
}

Received: 22 November 2018; Accepted: 21 December 2018; Published: 28 December 2018

check for updates

\begin{abstract}
An abdominal aortic aneurysm is a localized expansion of the abdominal aorta with a diameter $>3 \mathrm{~cm}$ or $>50 \%$ larger than the normal diameter. In this study, the stretch and strength of the materials in the abdominal aorta in patients with aneurysms were examined based on the results of tensile tests, and databases of failure stress and stretch were established according to age. Generally, the tensile test results of the axial and circumferential directions have become a priority in the tests of aortic materials. However, this study focused on the results of the axial direction. In addition, finite element analysis, where the Holzapfel model and the test results were applied, was performed. As a result, the behavior characteristics of the abdominal aortic materials were precisely simulated. The formula and material constants used in the Holzapfel model were studied and proposed in order to simulate the failure stress and stretch according to age as well as simulation.
\end{abstract}

Keywords: abdominal aorta aneurysm; anisotropic hyper-elastic model; material nonlinear behavior; finite element analysis

\section{Introduction}

The aorta is the main artery in the human body, originating from the left ventricle of the heart and extending down to the abdomen, where it splits into two smaller arteries. It distributes oxygenated blood to all parts of the body through systemic circulation. However, aortic aneurysms-characterized by reduced stiffness of the arterial wall-may develop, which results in the expansion of the lumen due to several factors, such as smoking, alcohol consumption, hypertension, genetic influences, and atherosclerosis. Aneurysms have different names according to their position, and their geometry is also different depending on the location. In particular, an abdominal aortic aneurysm is a localized enlargement of the abdominal aorta with a diameter $>3.0 \mathrm{~cm}$ or $50 \%$ larger than the normal diameter. 
These abdominal aortic aneurysms occur most commonly in individuals aged 50 years for men and 60 to 70 years for women, as well as those with family history [1]. Additional risk factors include smoking, high blood pressure, other heart or blood vessel diseases, and genetic conditions, including Marfan syndrome and Ehlers-Danlos syndrome, which increase risk [2]. According to the guidelines of the European Society for Vascular Surgery, it can be seen that important risk factors for abdominal aortic aneurysms are advanced age, male gender, and smoking. Smoking is a particularly strong risk factor, and smoking-related risks are much higher than for either coronary artery disease or stroke. In addition, it can be seen that the average growth rate of abdominal aortic aneurysms is from $30 \mathrm{~mm}$ to $55 \mathrm{~mm}$, and the ranges are from $2 \mathrm{~mm}$ to $3 \mathrm{~mm}$ per year [2]. In these abdominal aortic aneurysms, ruptures are very lethal and the mortality rate is from $85 \%$ to $90 \%$. In particular, the aneurysms are normally asymptomatic until a rupture. Hence, the aneurysms should be identified and treated before any ruptures occur, and surgery is usually recommended for abdominal aortic aneurysms with a diameter of $>5.5 \mathrm{~cm}$ in men or $>5.0 \mathrm{~cm}$ in women in order to prevent aortic ruptures [1].

However, there are some problems in the surgical judgement criteria for abdominal aortic aneurysms. In particular, many clinicians are having difficulty in examining the cause and making accurate judgements of aortic aneurysms, partly because aortic ruptures more commonly occur even if the diameter is $<5.0 \mathrm{~cm}$. Hence, studies on the risk factors of abdominal aortic aneurysms have been conducted in recent decades, and some studies reported the significant associations of these factors with the number of patients with abdominal aortic aneurysms. Nevertheless, studies that quantitatively analyze the location, propagation rate, and rupture risk of abdominal aortic aneurysms are rarely able to be found.

Therefore, the mechanical analysis in identifying the precise material behavior and degradation of abdominal aortic properties according to the major factors should be performed in order to solve these problems. Experimental and computational analyses of the extracted tissues from carcasses, cadavers, and patients have been conducted to identify the precise material behaviors and properties based on various factors, such as vascular location [3-7], layer [8-11], diameter [12], and smoking preference [5] in leading overseas research institutions for many decades [13-17].

In particular, Pierce et al. [3] have quantitatively studied the impact of tissue variations on the passive mechanical response of aneurysms in the supra-physiological loading range via mechanical testing and constitutive modeling. They conducted uniaxial extension tests on circumferentially and axially oriented strips from five thoracic and eight abdominal aortic fusiform aneurysms. As a result, they found the differences on the material behavior between the diseased thoracic and abdominal tissues and between healthy and diseased tissues. In addition, databases on the material parameters were presented using the Holzapfel model, a constitutive model that simulates the vessel, and an evaluation method of the damaged tissues, as well as the material nonlinear behavior were proposed. Forsell et al. [4] also assessed the risk of abdominal aortic aneurysm ruptures by using the tensile test and finite element methods, which analyzed the circumferentially and axially oriented strips from sixteen abdominal aortic aneurysms. They also proposed the elasto-plastic damage model to predict the failure stress and strain in the arterial wall. Raghavan et al. $[5,6]$ performed tensile tests for unruptured and ruptured abdominal aortic aneurysms, and examined wall thickness ranges according to aneurysm diameters. Moreover, they reviewed the failure stress, strain, and tension based on the location of the aneurysm, orientation of fiber, patient's age and smoking preference, and presented the average stress and strain data and relationship between stress and strain in healthy and diseased tissues. Pancheri et al. [7] provided new insights on the relationships between patients' natural histories, histopathologies, and mechanical behavior that may be useful in identifying the rupture risk evaluation method. Tong et al. [8] examined the biaxial extension behavior of the intraluminal thrombus and thrombus-covered wall in abdominal aortic aneurysms according to the patient's age, and compared this with the proposed material models. They measured the behaviors of the ruptured luminal, medial, and abluminal layers in the circumferential and longitudinal directions. Gasser et al. [9] proposed an in vitro experimental approach that provided layer-specific failure data of the 
intraluminal thrombus tissue under static and pulsatile mechanical loads. In addition, they presented various intraluminal thrombus ultimate strength and stiffness across the radial direction using data from the luminal, medial, and abluminal specimens.

Despite the performance of these various studies, some limitations, such as the age range, numerical model for material nonlinear-damage modeling, and criterion for rupture in abdominal aortic aneurysms are still observed. The material properties of the fiber direction in the abdominal aorta tend to decrease as age increases, and the tissues have material nonlinearities according to age [18]. Hence, in experimental and computational studies of the abdominal aorta, advanced studies that assess the variation of material properties according to age and develop a numerical model for material nonlinear-damage modeling should be conducted.

Therefore, this study examined the information, tensile tests, and numerical theories for abdominal aortic aneurysms, and the material behaviors of the tissues in patients with abdominal aortic aneurysms were simulated using the ABAQUS finite element program. In the simulation step, the Holzapfel model $[19,20]$ was used to analyze the material model. In addition, in order to determine the reliability of the simulation technique, the numerical simulations under uniaxial tensile loading for the axial and circumferential directions were compared with experimental results. The stress of the tissue was examined according to age from the numerical simulation and the experimental results. Consequently, the material constants of the failure stress and stretch of the tissues in patients with abdominal aortic aneurysms were proposed according to age. Furthermore, the relationship between material constants and age was examined, and the formula used to determine the relationship was also proposed. These results could be used to examine the wall stress of aortas in patients with abdominal aortic aneurysms, and the results are also expected to be used in the simulations that were conducted to predict rupture risks in abdominal aortic aneurysms.

\section{Methodology}

In this study, the failure stress and stretch according to age in the experimental results were examined, and the relationship between the results was investigated. The numerical model proposed by Holzapfel et al. [19-21] was used for the computational analysis method to simulate the experimental method.

\subsection{Experiment Details}

This study reviewed the experimental results of the tensile tests carried out by Pierce et al. [3] and Forsell et al. [4] under a temperature condition of $37^{\circ} \mathrm{C}$. Pierce et al. [3] conducted uniaxial tensile tests on circumferentially and axially oriented strips from eight abdominal aortic aneurysms (63.9 years \pm 11.4 ). They glued sandpaper to the ends of the samples in order to prevent slippage, and the specimens were obtained as rectangular strips $(4 \times 15 \mathrm{~mm}$, width $\times$ length). In addition, they conducted uniaxial testing under quasi-static conditions with a displacement rate of $5 \mathrm{~mm} / \mathrm{min}$ in order to avoid dynamic or viscoelastic effects. As a result, they examined the relationships between stress and stretch of tissue and investigated the material damage parameters. Forsell et al. [4] also performed the uniaxial tensile tests on longitudinally oriented strips from 14 abdominal aortic aneurysms (68.9 years \pm 6.7 ). They prepared the bone-shaped test specimens that measured $8 \times$ $36 \mathrm{~mm}$ (width $\times$ length) with $4 \mathrm{~mm}$ (width in the middle) in order to reduce the failure rate of the experiment. In addition, the specimens were also clamped at each end using the grips attached to the crossheads of the tensile test equipment and the stress-stretch curve for the tissues presented from the experimental results. In their study, the stretch, instead of strain, of the arterial tissue was examined in the experimental results under the tensile loading, because it is very difficult to measure the strain of arterial tissue using the video- or laser-extensometer in the specimens of small dimensions.

Consequently, the failure stress and stretch measurements obtained from the experimental results of individuals aged 58-78 years were presented in the megapascal scale, as shown in Figure 1. Samples clearly have failure stress values of approximately $0.11-0.79 \mathrm{MPa}$, and failure stretch values 
of approximately 1.08-1.60. Moreover, the trend line obtained based on the relationship between age and failure stress and stretch data were added in Figure 1, and the failure stress and stretch values based on this trend line were calculated to determine the material constants used in the numerical model during the computational simulation. In this study, the material behavior of the aorta in the abdominal aortic aneurysm was investigated in patients aged 58-78 years, because it most commonly occurs in those aged $>50$ years [1].

As a result, the failure stress and stretch in the aortic tissue appears to have an important effect on decreasing the tensile strength, as the age increased, and the trend in the database could be recognized. In addition, the minimum and maximum values of the trend were determined, and the criteria of the damage characteristics of aortic tissue in patients with abdominal aortic aneurysms could be established based on these trend lines. The material parameters in the numerical model that applies the computational simulation were calculated based on these experimental results.

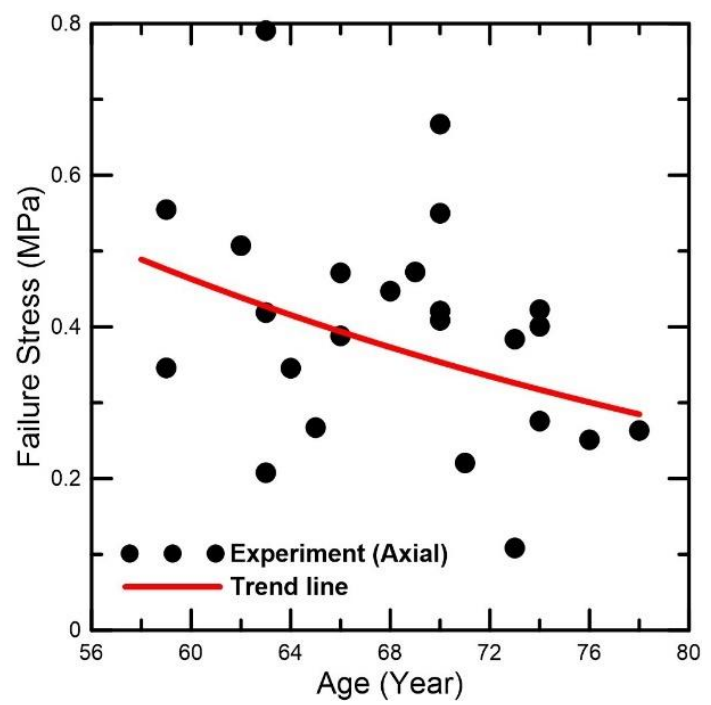

(a)

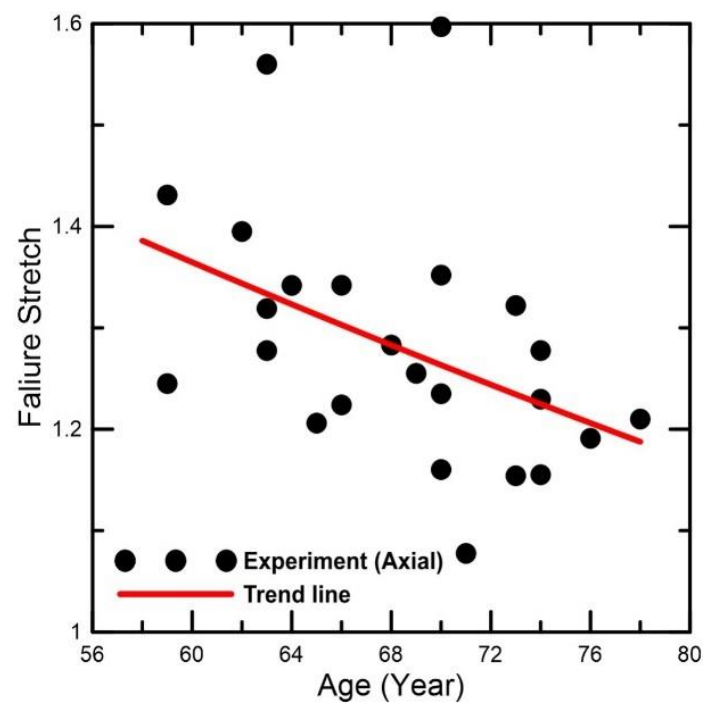

(b)

Figure 1. Failure (a) stress and (b) stretch distribution, according to age.

\subsection{Numerical Model}

Theories on anisotropic hyper-elastic material of the arterial layers were developed by many research teams and have been applied in the field of computational biomechanics [19-24]. In particular, the Fung [22] and Holzapfel $[19,20]$ models have been widely used in various simulations of the arterial wall. In the Fung model [22], the underlying assumption is based on a strain formulation that the preferred material directions are initially aligned with an orthogonal coordinate system in the reference configuration. These directions may become non-orthogonal only after the deformation. In the Holzapfel model $[19,20]$, unlike the strain-based formulation, the invariant-based formulation of fiber directions need not be orthogonal during the initial configuration. Therefore, a constitutive law on the description of mechanical responses in the arterial tissues has been proposed, and the arterial walls were considered as a thin-walled circular cylindrical tube consisting of layers.

In the present study, the Holzapfel model $[19,20]$ was applied to the numerical simulation, and the material constants according to age were calculated. Moreover, the formulae for material constants including the age were proposed.

Hyper-elastic materials. In hyper-elastic materials, the relationship between stress and strain are determined on the basis of the strain energy density function $(\psi)$, which is defined according to a 
deformation gradient or strain tensor. The derivative of the strain energy density function of a strain component provides its corresponding stress component [24]:

$$
S_{i j}=\frac{\partial \psi}{\partial \varepsilon_{i j}}
$$

where $\mathbf{S}$ is the second Piola-Kirchhoff stress tensor and $\varepsilon$ is the Lagrangian strain tensor, which is defined as follows:

$$
\varepsilon_{i j}=\frac{1}{2}\left(C_{i j}-\delta_{i j}\right)
$$

In Equation (2), $\mathbf{C}$ is the right Cauchy-Green deformation tensor $\left(\mathbf{C}=\mathbf{F}^{\mathrm{T}} \mathbf{F}\right)$, and $\mathbf{F}$ is the deformation gradient that can be presented in terms of the displacement vector $\mathbf{u}(\mathbf{F}=\nabla \mathbf{u}+\mathbf{I})$. Local geometry deformation of a material element is explained on the basis of the deformation gradient, and the strain energy function is commonly defined as a function of the deformation gradient. Actually, the function is expressed as the right Cauchy-Green deformation tensor. Therefore, the relationship of the second Piola-Kirchhoff with the Cauchy stress tensor is expressed as follows [24]:

$$
\mathbf{S}=J \mathbf{F}^{-1} \sigma \mathbf{F}^{-T}
$$

where $J=\operatorname{det} \mathbf{F}$. Therefore, the Cauchy stress tensor is defined according to the following Equations (1)-(3):

$$
\sigma=2 J^{-1} \mathbf{F} \frac{\partial \psi}{\partial \mathbf{C}} \mathbf{F}^{T}
$$

In isotropic materials, the strain energy density function is dependent on the right Cauchy-Green deformation tensor based on its invariants. The invariants of the right Cauchy-Green deformation tensor are as follows:

$$
I_{1}=\operatorname{trC} I_{2}=\frac{1}{2}\left[(\operatorname{tr} \mathbf{C})^{2}-\operatorname{trC}^{2}\right] \quad I_{3}=\operatorname{det} \mathrm{C}
$$

Therefore, the strain energy density function is dependent on the invariants [20], and the Cauchy stress equation is expanded as follows:

$$
\sigma=2 J^{-1}\left(\mathbf{F} \frac{\partial \psi}{\partial I_{1}} \frac{\partial I_{1}}{\partial \mathbf{C}} \mathbf{F}^{T}+\mathbf{F} \frac{\partial \psi}{\partial I_{2}} \frac{\partial I_{2}}{\partial \mathbf{C}} \mathbf{F}^{T}+\mathbf{F} \frac{\partial \psi}{\partial I_{3}} \frac{\partial I_{3}}{\partial \mathbf{C}} \mathbf{F}^{T}\right)
$$

By obtaining the derivatives of the invariants with respect to the right Cauchy-Green deformation tensor [24] and knowing that $\mathbf{B}=\mathbf{F F}^{\mathrm{T}}$, Equation (6) can be expressed as follows:

$$
\sigma=2 J^{-1}\left[\psi_{1} \mathbf{B}+\psi_{2}\left(I_{1} \mathbf{B}-\mathbf{B}^{2}\right)+I_{3} \psi_{3} \mathbf{I}\right]
$$

where $\psi=\partial \psi / \partial I_{i}$. In an incompressible isotropic material, $I_{3}=\operatorname{det} \mathbf{F}=1$, and the Cauchy stress tensor is modified as follows [24]:

$$
\sigma=-p \mathbf{I}+2 \mathbf{F} \frac{\partial \psi}{\partial \mathbf{C}} \mathbf{F}^{T}
$$

where $p$ is a scalar identified as hydrostatic pressure. Therefore, the Cauchy stress tensor of an incompressible material associated with $\mathbf{B}$ is expressed as follows:

$$
\sigma=-p \mathbf{I}+2 \psi_{1} \mathbf{B}+2 \psi_{2}\left(I_{1} \mathbf{B}-\mathbf{B}^{2}\right)
$$

Anisotropic hyper-elastic materials. In the organization of human soft tissues, the existence of collagen fibers [25] causes the material to have one or more preferred directions (M). In this case, the strain energy density is a function of both the right Cauchy-Green deformation tensor and 
the preferred direction. Two more dependent pseudo-invariants for these materials are defined as follows [19]:

$$
I_{4}=\mathbf{M}(\mathbf{C M}) \quad I_{5}=\mathbf{M}\left(\mathbf{C}^{2} \mathbf{M}\right)
$$

where, for example, $\mathbf{C M}$ expresses the action of the second-order tensor $\mathbf{C}$ on the vector $\mathbf{M}$.

In an incompressible material reinforced by one family of fibers, the strain energy density function is dependent on $I_{1}, I_{2}, I_{4}$, and $I_{5}$. In this particular case, the Cauchy stress has two additional terms that indicate the effects of anisotropy. Therefore, the Cauchy stress tensor could be represented as follows [20]:

$$
\sigma=-p \mathbf{I}+2 \psi_{1} \mathbf{B}+2 \psi_{2}\left(I_{1} \mathbf{B}-\mathbf{B}^{2}\right)+2 \psi_{4} \mathbf{m} \otimes \mathbf{m}+2 \psi_{5}[\mathbf{m} \otimes \mathbf{B m}+\mathbf{B m} \otimes \mathbf{m}]
$$

where $\otimes$ denotes the dyadic product of two vectors and $\mathbf{m}=\mathbf{F M}$ is the deformed form of the vector $\mathbf{M}$ in the present configuration. In some tissues, such as arterial walls, two families of fibers with different directions can be found within the tissue. Therefore, $\mathbf{M}^{\prime}$ can be considered as the unit vector in the direction of the second family of fibers. In addition, three more invariants were considered as follows [20]:

$$
I_{6}=\mathbf{M}^{\prime}\left(\mathbf{C M}^{\prime}\right) \quad I_{7}=\mathbf{M}^{\prime}\left(\mathbf{C}^{2} \mathbf{M}^{\prime}\right) \quad I_{8}=\left[\mathbf{M}\left(\mathbf{C M}^{\prime}\right)\right]\left(\mathbf{M M}^{\prime}\right)
$$

In this case, the Cauchy stress is represented as follows [20]:

$$
\begin{gathered}
\sigma=-p \mathbf{I}+2 \psi_{1} \mathbf{B}+2 \psi_{2}\left(I_{1} \mathbf{B}-\mathbf{B}^{2}\right)+2 \psi_{4} \mathbf{m} \otimes \mathbf{m}+2 \psi_{5}[\mathbf{m} \otimes \mathbf{B m}+\mathbf{B m} \otimes \mathbf{m}] \\
+2 \psi_{6} \mathbf{m}^{\prime} \otimes \mathbf{m}^{\prime}+2 \psi_{7}\left[\mathbf{m}^{\prime} \otimes \mathbf{B m}^{\prime}+\mathbf{B m}^{\prime} \otimes \mathbf{m}^{\prime}\right]+2 \psi_{8}\left(\mathbf{M} \otimes \mathbf{M}^{\prime}\right)\left(\mathbf{m} \otimes \mathbf{m}^{\prime}+\mathbf{m}^{\prime} \otimes \mathbf{m}\right)
\end{gathered}
$$

In this study, the Holzapfel model for anisotropic materials was used as the simulation method, and the strain energy function in the Holzapfel model is expressed as follows [20]:

$$
\psi=C_{10}\left(I_{1}-3\right)+\frac{k_{1}}{k_{2}}\left\{\exp \left[k_{2}\left(I_{4}^{*}-1\right)^{2}\right]-1\right\}
$$

where $C_{10}, k_{1}, k_{2}$, and $\kappa$ are material constants, and $I_{4}^{*}$ is described as follows:

$$
I_{4}^{*}=\kappa I_{1}+(1-3 \kappa) I_{4}
$$

In the Holzapfel model, the direction of each family of fibers is assumed to be dispersed in a mean direction. The dispersion is indicated based on $\kappa(0 \leq \kappa \leq 1 / 3)$ [20], and the Cauchy stress tensor could be calculated from Equations (13)-(15).

\subsection{Simulation Details}

The estimated failure stress and strain from the trend line determined in Section 2.1 were used to analyze the material constants. In this study, the ABAQUS finite element analysis program was used to compare the experimental results in the tensile test of the soft tissue. First, the material constants in the numerical model were calculated on the basis of the stress-stretch curve according to the orientation of tissues, in order to assure the reliability of the computational simulation technique. The material constants of the stress and stretch according to age were calculated using the previous computational simulation technique.

The geometry of the tensile specimen was set as rectangular strips $(4 \times 15 \times 2.12 \mathrm{~mm}$, width $\times$ length $\times$ thickness) based on the dimensions of the experiment in Section 2.1, as shown in Figure 2 . The orientations of the collagen fiber in the layers were set as 18.8, 37.8, and 58.9 degrees (Intima, Media, Adventitia), as shown in Figure 3 [21] and Figure 4. The measured specimens were embedded maintaining planar geometry and sectioned sequentially at $3 \mu \mathrm{m}$ in a tangential orientation, and the 
fiber orientations in the plane were observed on the histological images. In addition, the orientations for 60 collagen fibers were measured in the intima, adventitia, and oblate nuclei of smooth muscle cells in the media. Therefore, Holzapfel [21] proposed the determined mean fiber angles and standard deviations numerically by assuming a normal distribution and symmetrical arrangement in accordance with the circumferential direction.

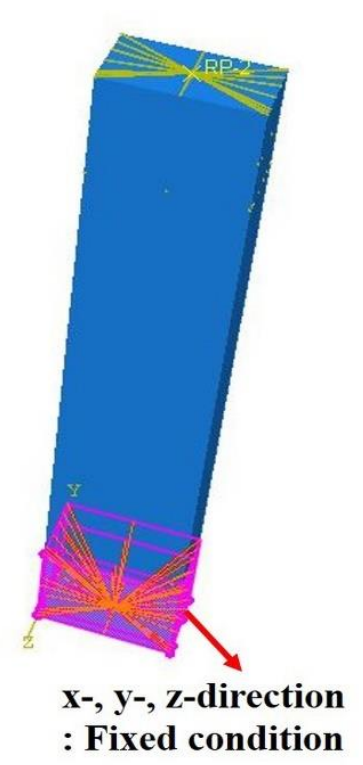

(a)

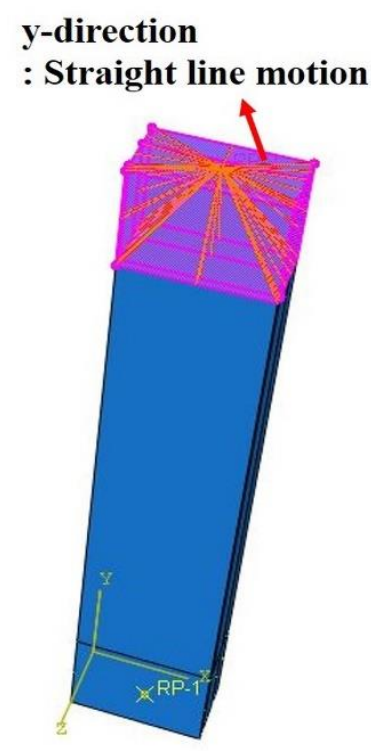

(b)

Figure 2. (a) Boundary and (b) loading conditions of the tensile test specimen during the simulation.
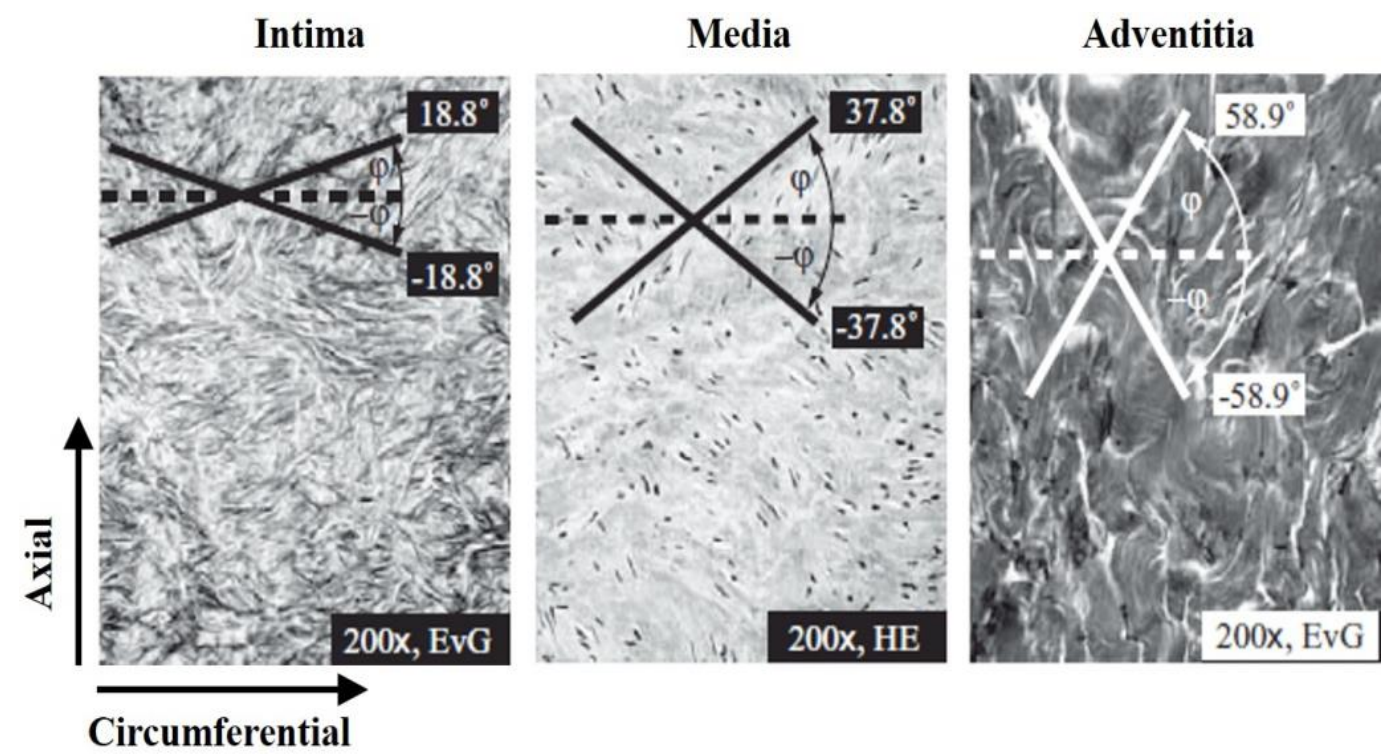

Figure 3. Images of an intimal, medial, and adventitial strip with circumferential orientation [21].

In the boundary and loading conditions, the fixed and straight-line motion conditions were applied in the experimental conditions, as shown in Figure 2. The fixed and straight-line motion conditions were applied at both ends of the specimen. Moreover, in the material properties, the Holzapfel model of the anisotropic hyper-elastic material was used for the simulation, and the element used was type C3D8RH, a general-purpose linear brick element with reduced integration, hourglass control, and hybrid with constant pressure [26]. In this simulation, the numerical analysis of 
the tensile tests for adventitial strips that cut along the circumferential directions of the artery, as well as the strip cut, was considered, as shown in Figure 4.

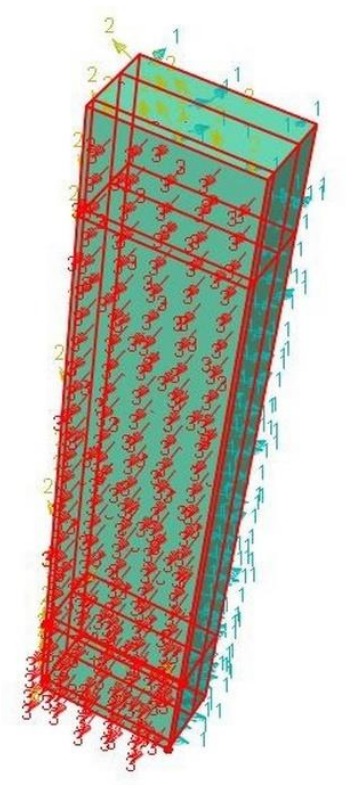

(a)

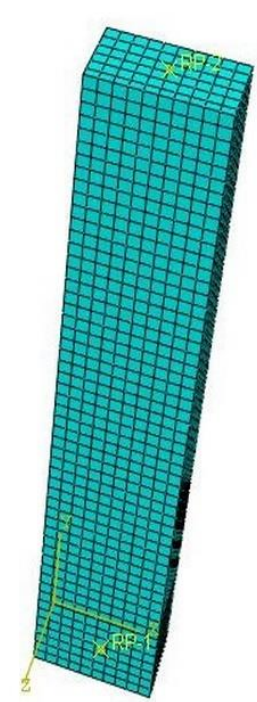

(b)

Figure 4. (a) Orientations of the fiber and (b) mesh in the tensile test specimen during the simulation.

\section{Results and Discussion}

\subsection{Tensile Strength of Arterial Tissues According to Fiber Orientation}

To demonstrate the reliability of the results in numerical analysis, simulation results have to be validated by comparing them with the experimental results. Therefore, in this simulation study, the material constants in the Holzapfel model [20] were firstly established based on the stress-stretch curve of the tissues in patients with abdominal aortic aneurysms. In particular, the stress-stretch curve of the tensile tests according to the fiber orientation was estimated from Pierce et al.'s experimental results [3] where the representative stress-stretch experimental data and equation fits for the tensile test data were provided. In addition, numerical simulations were conducted through a uniaxial extension simulation of circumferentially and axially oriented strips, as shown in Figure 5.

The arterial tissue has different tensile stress and stretch according to the direction, because they are composed of three layers that have different fiber directions according to the layer. Therefore, the samples on the circumferentially oriented strip had an average tensile strength value of 0.34 $\mathrm{MPa}$, which is $14.3 \%$ higher than the average value of the samples on the axially oriented strip. On the contrary, in the stretch results, the samples on the axially oriented strip had an average tensile stretch value of 1.23 , which is $3.0 \%$ higher than the average value of the samples on the circumferentially oriented strip. Therefore, it appears that the arterial tissue has different tensile characteristics according to the fiber orientation. Figure 5 shows the verification for the material parameters based on the directions of fiber in the parametric simulation. Consequently, it can be seen that the correlation coefficients, $r$, were 0.9977 and 0.9986 in the comparison between the experimental and simulated results on the circumferentially oriented strip. In addition, the R-squared, as well as the correlation coefficients were examined, and they had R-squared values of 0.9954 and 0.9971 on the axially oriented strip. 


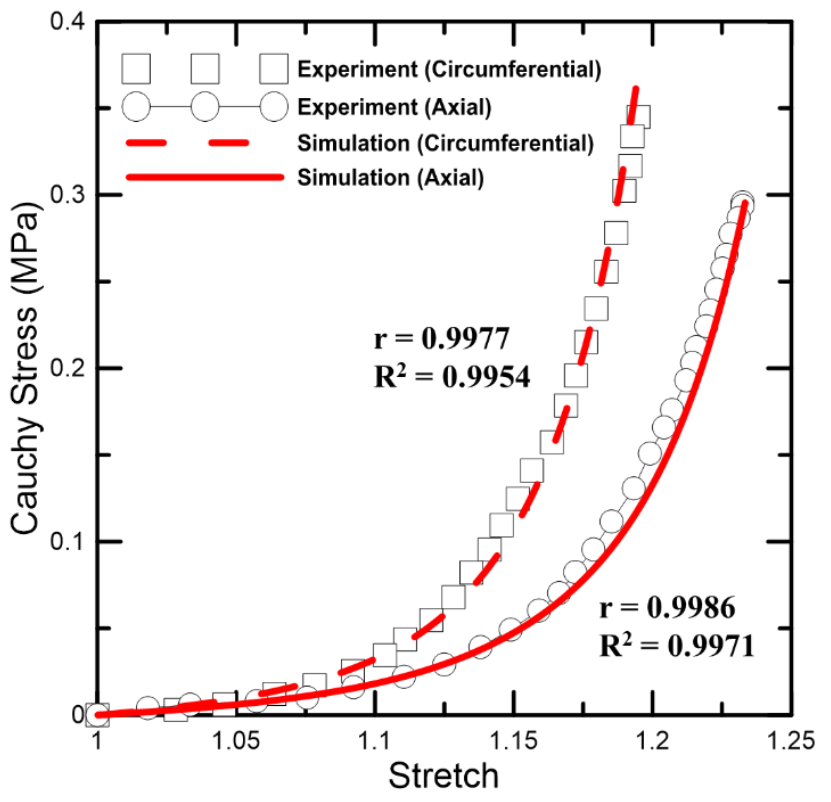

Figure 5. Stress-stretch curve of the arterial tissue determined during tensile tests and simulation.

\subsection{Tensile Strength of the Arterial Tissue According to Age}

In this study, the trend lines of the average, maximum, minimum values were calculated on the basis of the experimental results, and the simulation results under tensile loading for axial direction were compared with the experimental results according to age, as shown in Figure 6. Regarding the correlation between the trend lines of the maximum and minimum values and simulation based on the experimental results, a maximum error rate of 0.0065 and 0.0129 and an average error rate of 0.0021 and 0.0031 , respectively, were clearly observed. In addition, according to the line graph, the range of the failure stress at the age of 58 years is $0.274-0.704 \mathrm{MPa}$, and the range of the failure stretch is $1.216-1.556$. The range of the failure stress at the age of 78 years is $0.07-0.5 \mathrm{MPa}$, which is $29.0-74.5 \%$ lower than the failure stress at the age of 58 years, and the range for the failure stretch is 1.018-1.358, which is $12.7-16.3 \%$ lower than the failure stretch.

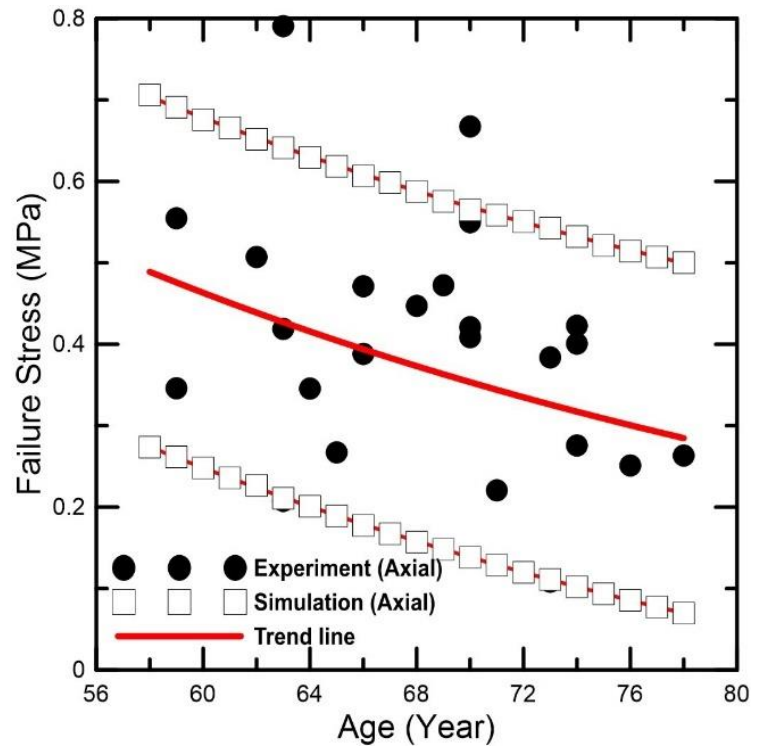

(a)

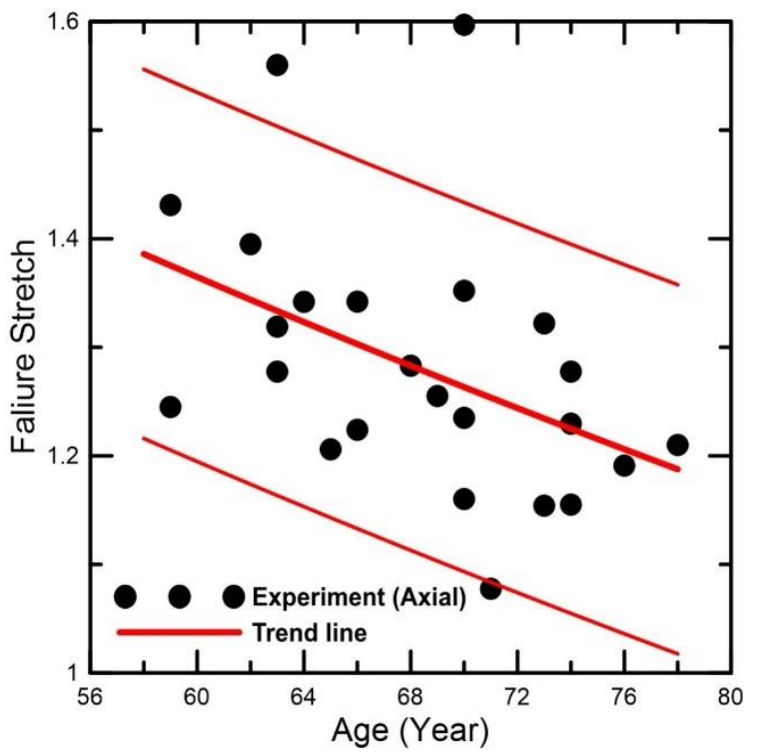

(b)

Figure 6. Experimental and simulation results, trend lines for (a) failure stress and (b) stretch. 
The material parameters, that were estimated from the simulation results under the axial tensile loading, were applied to the simulations according to age under the tensile loading for the circumferential direction, and the given line graph compares the tensile strength based on the arterial tissue samples of two different types of tensile loading directions in megapascal scale at ages 58-78 years, as shown in Figure 7. The range of the failure stress at the age of 58 years is clearly $0.182-0.839$ MPa on the circumferentially oriented strip, which is $19.9-73.6 \%$ lower than the failure stress at the age of 78 years. Notably, in the results of samples in patients aged $>76$ years, the failure stress on circumferentially oriented strip rapidly decreased from $0.182 \mathrm{MPa}$ to $0.304 \mathrm{MPa}$, although the arterial tissue has strong behavioral characteristics on the circumferentially oriented strip.

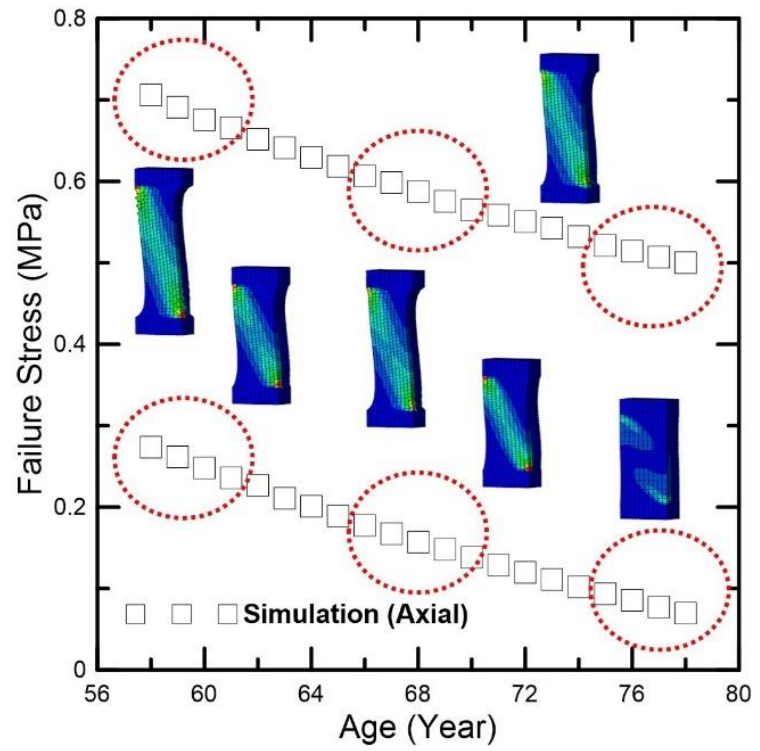

(a)

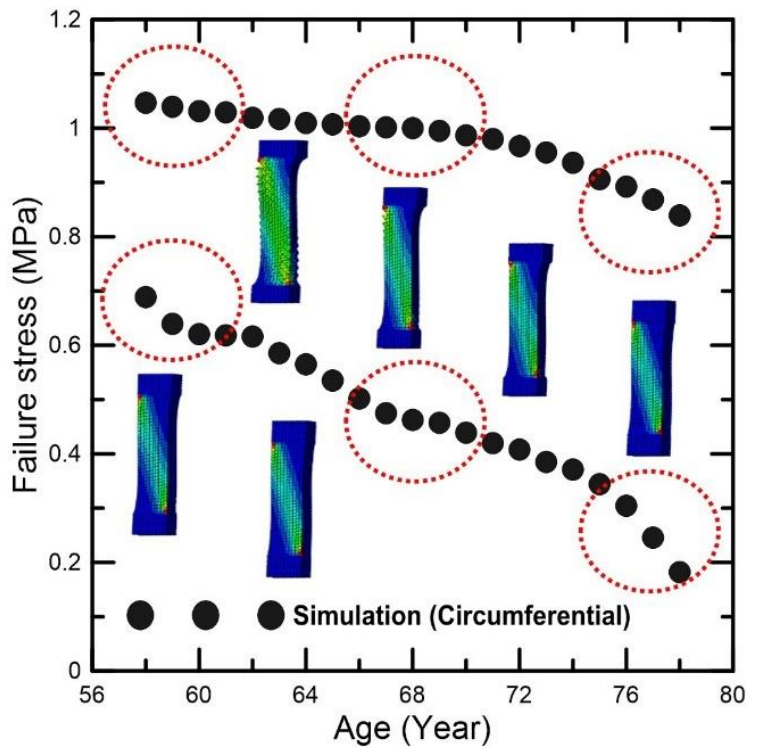

(b)

Figure 7. Simulation results under tensile loading for (a) axial and (b) circumferential directions.

\subsection{Parametric Study for Material Parameters}

In this parametric study, the material constants, $C_{10}, k_{1}$, and $k_{2}$, which are directly related to the young's modulus, stress, and strain of the material, respectively, were investigated. Figure 8 shows the variation of the material parameters according to age in the simulation results for the maximum and minimum trend line range. Consequently, the material constants on the maximum and minimum trend lines continuously increased overall as the age of the specimen increased. According to the line graph, the values of the material constant, $C_{10}$, increased sharply from 0.055 to 0.01 and from 0.353 to 0.02 on maximum and minimum trend lines, respectively, as age increased from 58 to 89 years. In addition, the values of the material constant, $k_{1}$, were nonlinearly related with age and increased from 6.5 to 580 on the minimum trend line, whereas the values of the material constant linearly increased from 0.87 to 1.84 on the maximum trend line. Finally, the values for the material constant, $k_{2}$, are also nonlinearly related with age and increased from 6.0 to 17.9 on the minimum trend line, whereas they are linearly related with age and increased from 63 to 355 on the maximum trend line. In addition, the material constants in the minimum trend line were higher than in the maximum trend line. This may have been due to the large deformation that occurs in the lower failure stress and stretch values, which sharply decreased as the age increased. 


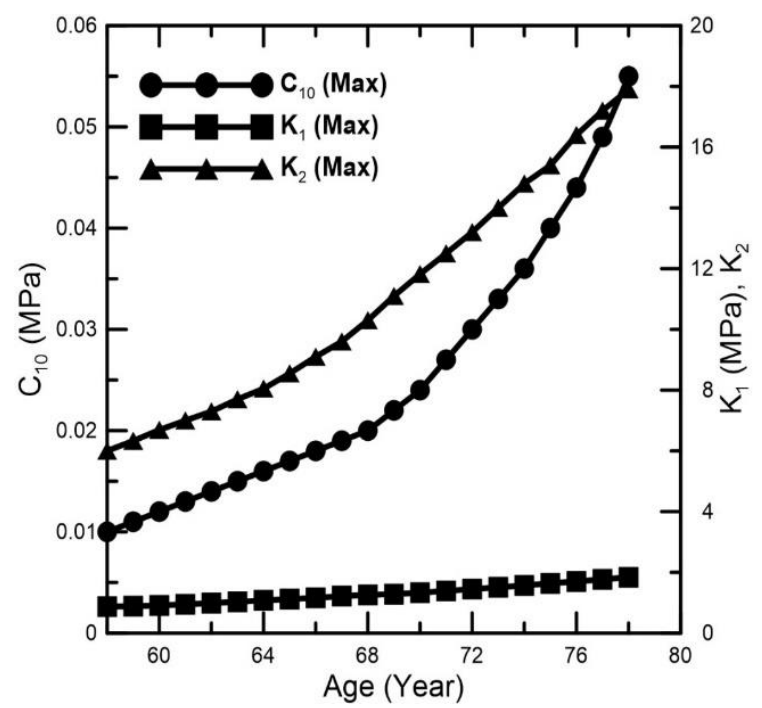

(a)

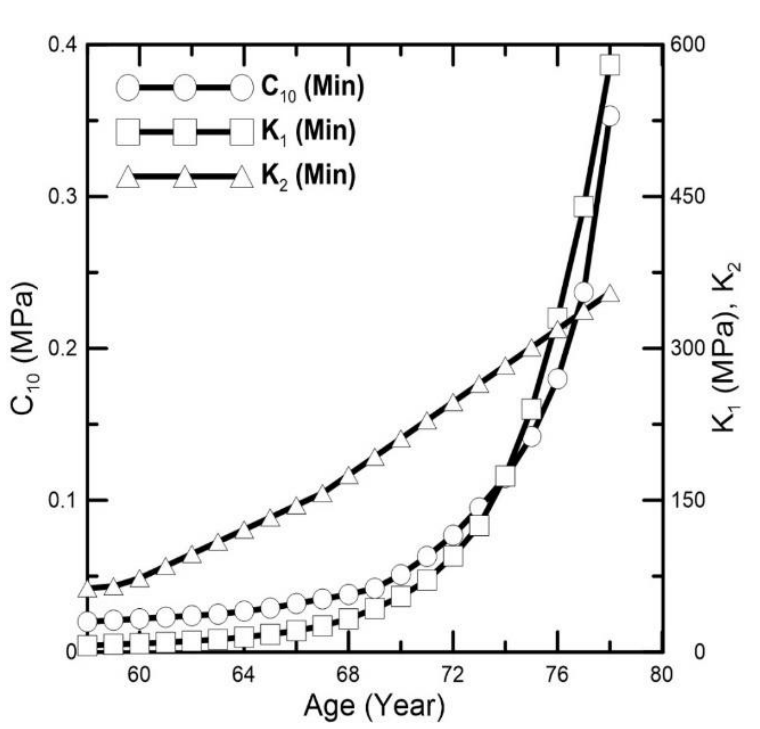

(b)

Figure 8. Variation of the material parameters according to age in the trend line range ((a) maximum and (b) minimum).

Moreover, the formulas in this study which can estimate the values of material constant associated with age $(N)$ shown in Figure 9, could be proposed as follows:

$$
\begin{gathered}
C_{10, \text { max }}=\alpha_{1, \text { max }} \times \exp \left(\alpha_{2, \text { max }} \times N\right) C_{10, \text { min }}=\alpha_{1, \text { min }} \times \exp \left(\alpha_{2, \text { min }} \times N\right) \\
k_{1, \text { max }}=\beta_{1, \text { max }} \times N-\beta_{2, \text { max }} k_{1, \text { min }}=\beta_{1, \text { min }} \times \exp \left(\beta_{2, \text { min }} \times N\right) \\
k_{2, \text { max }}=\gamma_{1, \text { max }} \times \exp \left(\gamma_{2, \text { max }} \times N\right) k_{2, \text { min }}=\gamma_{1, \text { min }} \times N-\gamma_{2, \text { min }}
\end{gathered}
$$

Figure 9 and Table 1 show the coefficients in Equations (16)-(18), and the comparison between material constants and formulae according to age. The R-squared is the proportion of variance in the dependent variable that can be distinguished from the independent variable. As a result, the R-squared ranges from 0.9152 to 0.9979 in the correlation between the values of the material constant and formulae. Therefore, the coefficients according to age can be calculated and predicted, and the simulation can be conducted in patients aged $>78$ years.

Table 1. Coefficients and R-squared in the correlation between values of material constants and formulae.

\begin{tabular}{cccc}
\hline Material Constant & $\boldsymbol{\alpha}_{1}, \boldsymbol{\beta}_{1}, \gamma_{1}$ & $\boldsymbol{\alpha}_{2}, \boldsymbol{\beta}_{2}, \gamma_{2}$ & $\boldsymbol{R}^{2}$ \\
\hline$C_{10, \max }$ & $8.4618 \mathrm{E}-05$ & 0.0818 & 0.9895 \\
$C_{10, \min }$ & $5.4244 \mathrm{E}-06$ & 0.1348 & 0.9152 \\
$k_{1, \text { max }}$ & 0.0485 & 2.0140 & 0.9863 \\
$k_{1, \text { min }}$ & $7.9912 \mathrm{E}-05$ & 0.2278 & 0.9760 \\
$k_{2, \max }$ & 0.2200 & 0.0567 & 0.9979 \\
$k_{2, \text { min }}$ & 15.2 & 844.9 & 0.9868 \\
\hline
\end{tabular}




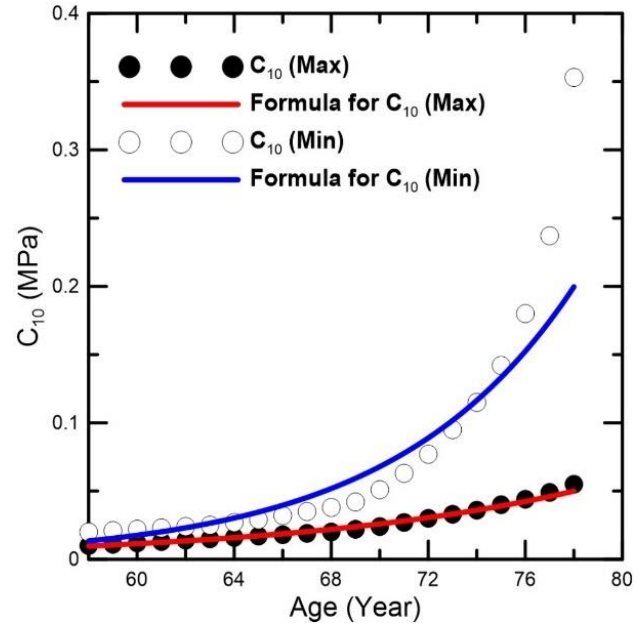

(a)

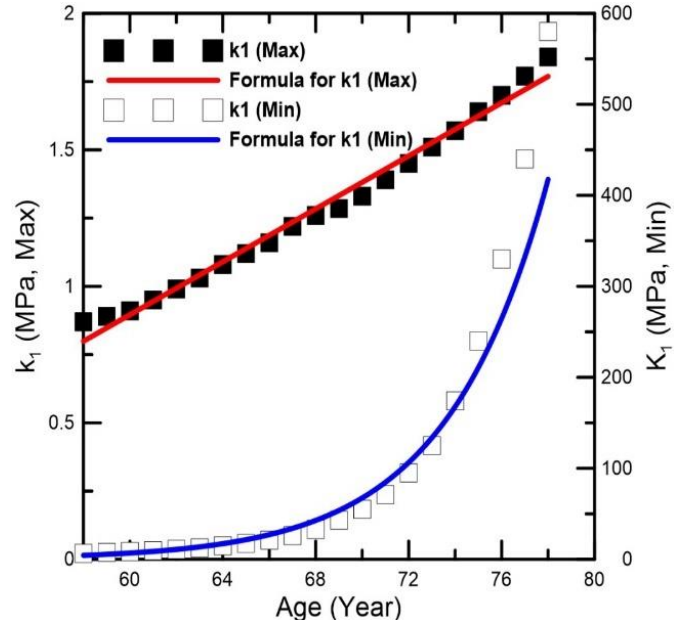

(b)

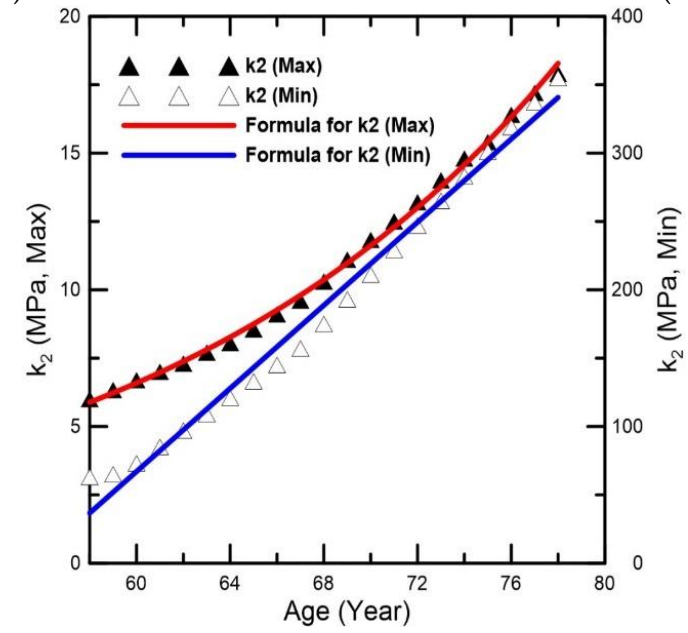

(c)

Figure 9. Comparison between material constants $\left((\mathbf{a}) C_{10},(\mathbf{b}) k_{1}\right.$, and (c) $\left.k_{2}\right)$ and formulae according to age.

\section{Conclusions}

This study investigated the uniaxial tensile tests and numerical theories of the samples extracted from the abdominal aorta in order to determine the material constants according to age in the numerical model. In addition, the numerical simulations were conducted under tensile loading for the axial and circumferential directions to observe the stress-stretch curve of the specimens, and the results were compared with experimental results to determine the reliability of the simulation method. From the numerical simulation and the experimental results, the stress of the specimen was examined according to age as well as the stress-stretch curve related to loading direction. Furthermore, the material parameters were determined according to age, and the formulae used in the material constants according to age were suggested. The results have the following general features:

- According to the tensile test results with respect to direction, the samples on the circumferentially oriented strip was noted to have an average tensile strength value of $0.34 \mathrm{MPa}$, which is $14.3 \%$ higher than that on the axially oriented strip. In addition, from the stretch results, it can be seen that the sample on the axially oriented strip had more flexibility than the sample on the circumferentially oriented strip. Therefore, the arterial tissue can be confirmed to have different tensile stress and stretch depending on the fiber orientation.

- Moreover, in this study, the failure stress and stretch from the uniaxial tensile test were examined according to age, and the trend lines of the average, maximum, and minimum values were 
calculated based on the experimental results. The numerical simulations according to age were conducted based on the results for the trend lines. As a result, a maximum error rate of 0.0065 and 0.0129 and an average error rate of 0.0021 and 0.0031 were observed in the comparison study between the trend lines and the simulations.

- In the parametric study, the material constants for age were estimated on the basis of the maximum and minimum trend lines. Regarding the correlation between material constants and age, they continuously increased overall as the age of the specimen increased. In particular, it can be seen that the values of the material constants, $C_{10}, k_{1, \min }$, and $k_{2, \max }$, were nonlinearly related with age.

- From these results, the formulae that associated the value of the material constant with age were proposed, and the proportion variance in the dependent variable that can be distinguished from the independent variable was examined. Consequently, it could be seen that the R-squared ranged from 0.9152 to 0.9979 in the correlation between the values of the material constant and the formulae.

The results obtained in the present study are expected to be used in the design for the medical device in order to enhance further understanding of the soft tissue behaviors. In addition, based on the results obtained in this study, we propose that the solid mechanics of the soft tissue must be considered when choosing the medical device design.

Author Contributions: Conceptualization: C.-W.L., C.S.L., J.-P.W. and D.-M.R.; methodology: U.H., C.-S.L., J.-P.W. and D.-M.R.; software: J.-H.Y., K.-H.K., C.-H.S. and D.-M.R.; validation: C.-W.L., J.-H.Y., C.-S.L. and D.-M.R.; formal analysis: U.H., K.-H.K. and J.-P.W.; investigation: C.-W.L., U.H., J.-H.Y., K.-H.K. and D.-M.R.; writing-original draft preparation: J.-H.Y., C.-S.L. and D.-M.R.; writing-review and editing: C.-W.L., U.H., C.-H.S., J.-P.W. and D.-M.R.

Funding: This work was supported by the National Research Foundation of Korea (NRF) grant funded by the Korea government (MSIT) (No. 2018R1C1B6007145). This research was supported by Basic Science Research Program through the National Research Foundation of Korea (NRF) funded by the Ministry of Education (2018R1D1A1B07044567).

Acknowledgments: In this section you can acknowledge any support given which is not covered by the author contribution or funding sections. This may include administrative and technical support, or donations in kind (e.g., materials used for experiments).

Conflicts of Interest: The authors declare no conflict of interest.

\section{References}

1. Kent, K.C. Abdominal aortic aneurysms. New Engl. J. Med. 2014, 371, 2101-2108. [CrossRef] [PubMed]

2. Moll, F.L.; Powell, J.T.; Fraedrich, G.; Verzini, F.; Haulon, S.; Waltham, M.; van Herwaarden, J.A.; Holt, P.J.E.; van Keulen, J.W.; Rantner, B.; et al. Management of abdominal aortic aneurysms clinical practice guidelines of the European society for vascular surgery. Eur. J. Vasc. Endovasc. Surg. 2011, 41, S1-S58. [CrossRef] [PubMed]

3. Pierce, D.M.; Maier, F.; Weisbecker, H.; Viertler, C.; Verbrugghe, P.; Famaey, N.; Fourneau, I.; Herijgers, P.; Holzapfel, G.A. Human thoracic and abdominal aortic aneurysmal tissues: Damage experiments, statistical analysis and constitutive modeling. J. Mech. Behav. Biomed. 2015, 41, 92-107. [CrossRef] [PubMed]

4. Forsell, C.; Swedenborg, J.; Roy, J.; Gasser, T.C. The quasi-static failure properties of the abdominal aortic aneurysm wall estimated by a mixed experimental-numerical approach. Ann. Biomed. Eng. 2013, 41, 1554-1566. [CrossRef]

5. Raghavan, M.L.; Hanaoka, M.M.; Kratzberg, J.A.; Higuchi, M.L.; Silva, E.S. Biomechanical failure properties and microstructural content of ruptured and unruptured abdominal aortic aneurysms. J. Biomech. 2011, 44, 2501-2507. [CrossRef] [PubMed]

6. Raghavan, M.L.; Kratzberg, J.; Tolosa, E.M.C.; Hanaoka, M.M.; Walker, P.; Silva, E.S. Regional distribution of wall thickness and failure properties of human abdominal aortic aneurysm. J. Biomech. 2006, 39, 3010-3016. [CrossRef] [PubMed] 
7. Pancheri, F.Q.; Peattie, R.A.; Reddy, N.D.; Ahamed, T.; Lin, W.; Ouellette, T.D.; Iafrati, M.D.; Dorfmann, A.L. Histology and biaxial mechanical behavior of abdominal aortic aneurysm tissue samples. J. Biomech. Eng. 2017, 139, 031002. [CrossRef] [PubMed]

8. Tong, J.; Cohnert, T.; Regitnig, P.; Holzapfel, G.A. Effects of age on the elastic properties of the intraluminal thrombus and the thrombus-covered wall in abdominal aortic aneurysms: Biaxial extension behaviour and material modelling. Eur. J. Vasc. Endovasc. Surg. 2011, 42, 207-219. [CrossRef]

9. Gasser, T.C.; Görgülü, G.; Folkesson, M.; Swedenborg, J. Failure properties of intraluminal thrombus in abdominal aortic aneurysm under static and pulsating mechanical loads. J. Vasc. Surg. 2008, 48, 179-188. [CrossRef]

10. Fereidoonnezhad, B.; Naghdabadi, R.; Holzapfel, G.A. Stress softening and permanent deformation in human aortas: Continuum and computational modeling with application to arterial clamping. J. Mech. Behav. Biomed. 2016, 61, 600-616. [CrossRef]

11. Wang, D.H.J.; Makaroun, M.; Webster, M.W.; Vorp, D.A. Mechanical properties and microstructure of intraluminal thrombus from abdominal aortic aneurysm. J. Biomech. Eng. 2001, 123, 536-539. [PubMed]

12. Tong, J.; Cohnert, T.; Holzapfel, G.A. Diameter-related variations of geometrical, mechanical, and mass fraction data in the anterior portion of abdominal aortic aneurysms. Eur. J. Vasc. Endovasc. Surg. 2015, 49, 262-270. [CrossRef] [PubMed]

13. Raghavan, M.L.; Webster, M.W.; Vorp, D.A. Ex vivo biomechanical behavior of abdominal aortic aneurysm: Assessment using a new mathematical model. Ann. Biomed. Eng. 1996, 24, 573-582. [CrossRef] [PubMed]

14. Niestrawska, J.A.; Viertler, C.; Regitnig, P.; Cohnert, T.U.; Sommer, G.; Holzapfel, G.A. Microstructure and mechanics of healthy and aneurysmatic abdominal aortas: Experimental analysis and modelling. J. R. Soc. Interface 2016, 13, 20160620. [CrossRef] [PubMed]

15. Polzer, S.; Gasser, T.C.; Bursa, J.; Staffa, R.; Vlachovsky, R.; Man, V.; Skacel, P. Importance of material model in wall stress prediction in abdominal aortic aneurysms. Med. Eng. Phys. 2013, 35, 1282-1289. [CrossRef] [PubMed]

16. Legerer, C.; Almsherqi, Z.A.; McLachlan, C.S. Over-Wrapping of the Aortic Wall with an Elastic Extra-Aortic Wrap Results in Luminal Creasing. J. Cardiovasc. Dev. Dis. 2018, 5, 42. [CrossRef] [PubMed]

17. Polanczyk, A.; Piechota-Polanczyk, A.; Domenig, C.; Nanobachvili, J.; Huk, I.; Neumayer, C. Computational Fluid Dynamic Accuracy in Mimicking Changes in Blood Hemodynamics in Patients with Acute Type IIIb Aortic Dissection Treated with TEVAR. Appl. Sci. 2018, 8, 1309. [CrossRef]

18. Ninomiya, O.H.; Monteiro, J.A.T.; Higuchi, M.L.; Puech-Leäo, P.; Luccia, N.; Raghavan, M.L.; Silva, E.S. Biomechanical properties and microstructural analysis of the human nonaneurysmal aorta as a function of age, gender and location: An autopsy study. J. Vasc. Res. 2015, 52, 257-264. [CrossRef]

19. Holzapfel, G.A.; Gasser, T.C.; Ogden, R.W. A new constitutive framework for arterial wall mechanics and a comparative study of material models. J. Elast. Phys. Sci. Solids 2000, 61, 1-48.

20. Holzapfel, G.A.; Ogden, R.W. Constitutive modelling of arteries. Proc. R. Soc. A Math. Phys. Eng. 2010, 466, 1551-1597. [CrossRef]

21. Holzapfel, G.A. Arterial tissue in health and disease: Experimental data, collagen-based modeling and simulation, including aortic dissection. In Biomechanical Modelling at the Molecular, Cellular and Tissue Levels; Holzapfel, G.A., Ogden, R.W., Eds.; Springer: Vienna, Austria, 2009; pp. 259-344. ISBN 978-3-211-95873-5.

22. Fung, Y.C.; Fronek, K.; Patitucci, P. Pseudoelasticity of arteries and the choice of its mathematical expression. Am. J. Physiol. 1979, 237, H620-H631. [CrossRef] [PubMed]

23. Humphrey, J.D. Mechanics of the arterial wall: Review and directions. Crit. Rev. Biomed. Eng. 1995, $23,1-162$. [CrossRef] [PubMed]

24. Holzapfel, G.A. Nonlinear Solid Mechanics; John Wiley and Sons Ltd.: Chichester, England, UK, 2000; pp. 205-357, ISBN 978-0-471-82319-3.

25. Unnikrishnan, V.U.; Unnikrishnan, G.U.; Reddy, J.N. Biomechanics of breast tumor: Effect of collagen and tissue density. Int. J. Mech. Mater. Des. 2012, 8, 257-267. [CrossRef]

26. ABAQUS. ABAQUS User's Manual; Dassault Systems: Vélizy-Villacoublay, France, 2014.

(C) 2018 by the authors. Licensee MDPI, Basel, Switzerland. This article is an open access article distributed under the terms and conditions of the Creative Commons Attribution (CC BY) license (http:/ / creativecommons.org/licenses/by/4.0/). 